\title{
Corrigendum to "A CDT-Based Heuristic Zone Design Approach for Economic Census Investigators"
}

\author{
Changxiu Cheng, ${ }^{1,2}$ Xiaomei Song, ${ }^{2,3}$ Jing Yang, ${ }^{1}$ Xiatian Hu, ${ }^{2}$ Shi Shen, ${ }^{1}$ and Lijun Wang ${ }^{4}$ \\ ${ }^{1}$ ADREM, Beijing Normal University, Beijing 100875, China \\ ${ }^{2}$ LREIS, Institute of Geographic Science and Resources Research, Beijing 100101, China \\ ${ }^{3}$ School of Software, Tsinghua University, Beijing 100084, China \\ ${ }^{4}$ China Internet Network Information Center, Beijing 100101, China \\ Correspondence should be addressed to Changxiu Cheng; chengcx@bnu.edu.cn
}

Received 31 December 2015; Accepted 3 January 2016

Copyright (C) 2016 Changxiu Cheng et al. This is an open access article distributed under the Creative Commons Attribution License, which permits unrestricted use, distribution, and reproduction in any medium, provided the original work is properly cited.

Our paper titled "A CDT-Based Heuristic Zone Design Approach for Economic Census Investigators” [1] contains an error in the author's name. The first author was incorrectly written as Changixu Cheng while the correct name is Changxiu Cheng. So the correct author list should be "Changxiu Cheng, Xiaomei Song, Jing Yang, Xiatian Hu, Shi Shen, and Lijun Wang," as shown above.

\section{References}

[1] C. Cheng, X. Song, J. Yang, X. Hu, S. Shen, and L. Wang, "A CDT-based heuristic zone design approach for economic census investigators," Mathematical Problems in Engineering, vol. 2015, Article ID 828374, 14 pages, 2015. 


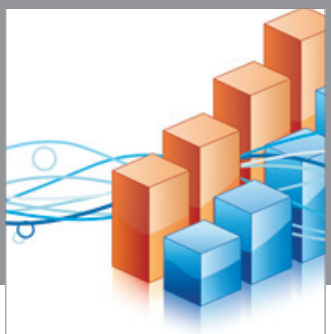

Advances in

Operations Research

vatem alat4

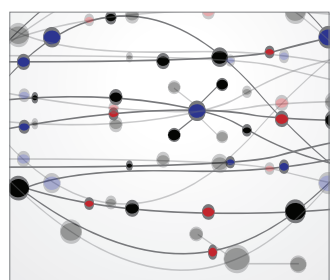

\section{The Scientific} World Journal
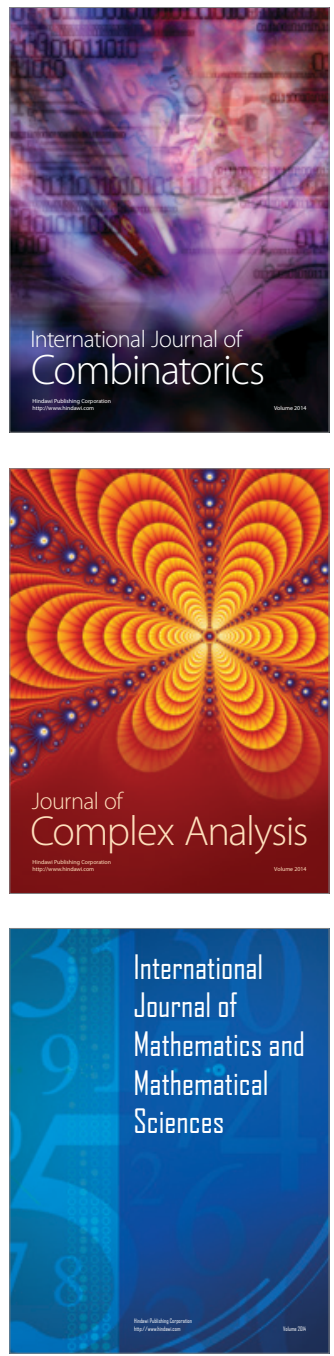
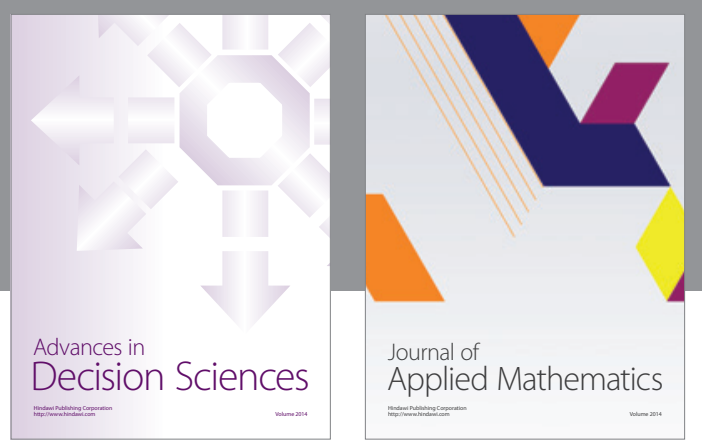

Algebra

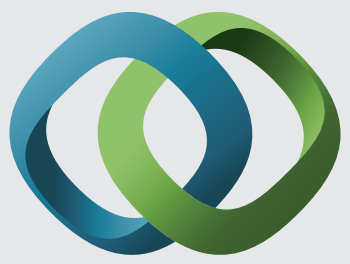

\section{Hindawi}

Submit your manuscripts at

http://www.hindawi.com
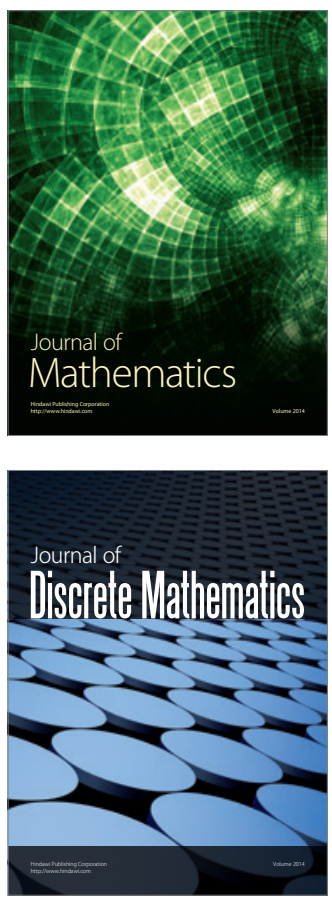

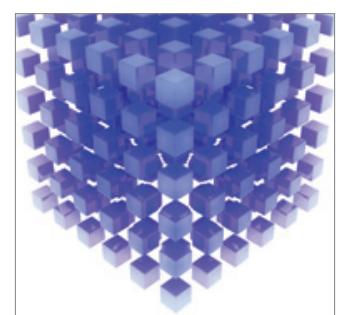

Mathematical Problems in Engineering
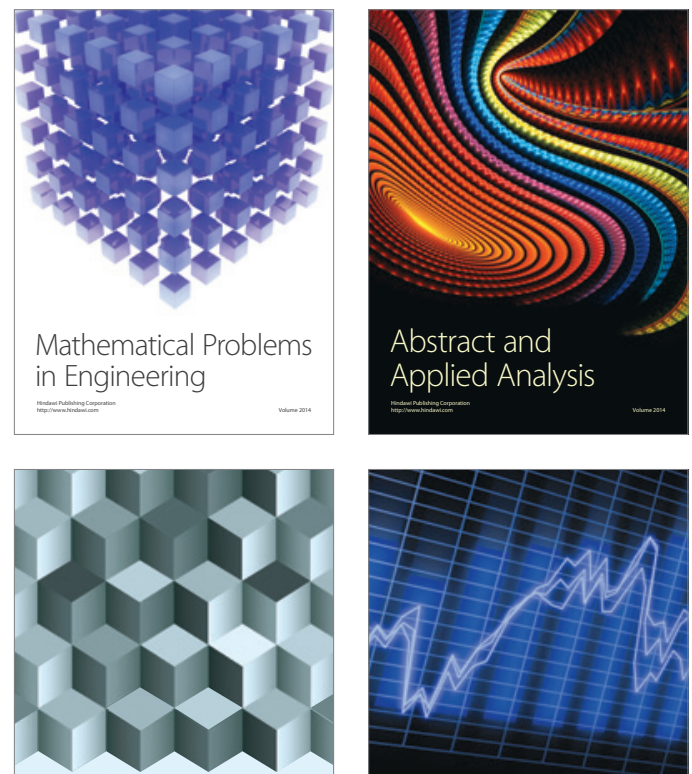

Journal of

Function Spaces

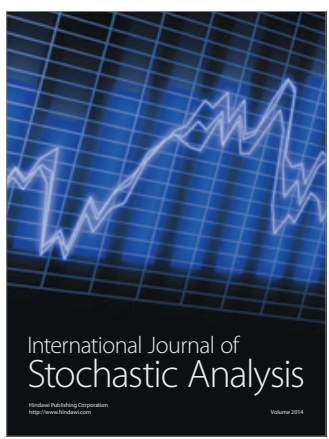

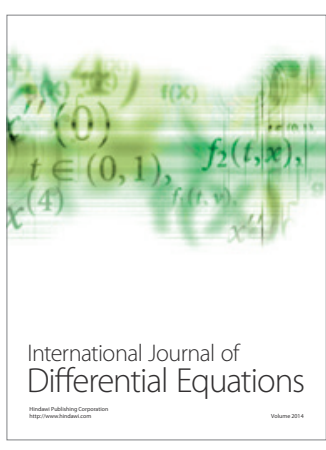
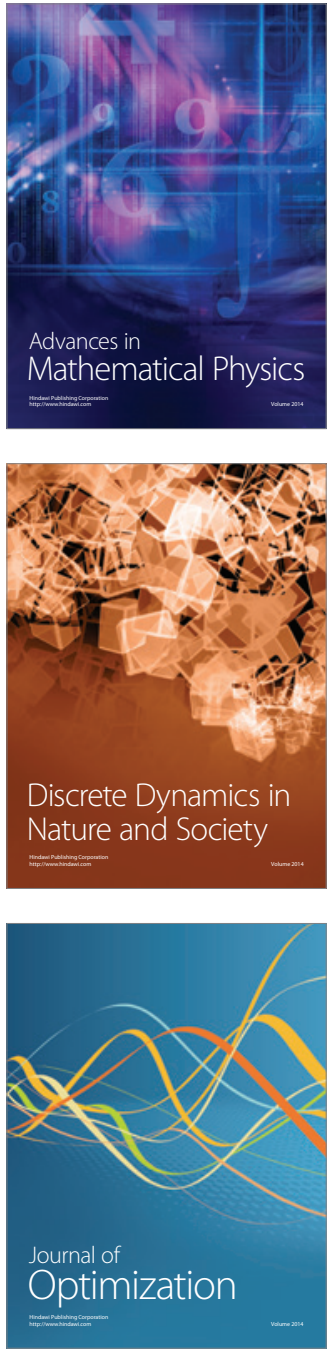